\title{
Novos Registros da família Aeshnidae (Odonata: Anisoptera) para o estado do Amapá, Brasil
}

A ordem Odonata com aproximadamente 7.000 espécies descritas mundialmente compreende o segundo maior grupo de insetos aquáticos. No Brasil, a ordem é representada por cerca de 860 espécies. Aeshnidae é uma família da subordem Anisoptera com ampla distribuição mundial, para o Brasil são conhecidas em torno de 60 espécies, sendo que, em muitos estados do país pouco se conhece sobre a família. Este trabalho, objetiva apresentar os dados de coletas realizadas durante o ano de 2018 em quatro municípios do estado do Amapá: Laranjal do Jari, Macapá, Porto Grande e Santana. Foram amostrados 58 exemplares de sete espécies incluídas em cinco gêneros pertencentes à família Aeshnidae. As espécies: Gynacantha nervosa Rambur, 1842, Neuraeschna costalis Burmeister, 1839, Staurophlebia reticulata (Burmeister, 1839), Triacanthagyna ditzleri Williamson, 1923 e Triacanthagyna septima (Selys in Sagra, 1857) contam como novos registros para o estado.

Palavras-chave: Diversidade; Amazônia; Fauna.

\section{New records of family Aeshnidae (Odonata: Anisoptera) for the state of Amapá, Brazil}

\begin{abstract}
The order Odonata with approximately 7,000 species described worldwide comprises the second largest group of aquatic insects. In Brazil, the order is represented by about 860 species. Aeshnidae is a family of the suborder Anisoptera with wide worldwide distribution, for Brazil they are known around of 60 species, being that, in many states in the country little is known about the family. This work aims to present data collected during 2018 in four municipalities in the state of Amapá: Laranjal do Jari, Macapá, Porto Grande and Santana. Were sampled 58 specimens of seven species were included, included in five genera belonging to the family Aeshnidae.The species: Gynacantha nervosa Rambur, 1842, Neuraeschna costalis Burmeister, 1839, Staurophlebia reticulata (Burmeister, 1839) Triacanthagyna ditzleri Williamson, 1923 and Triacanthagyna septima (Selys in Sagra, 1857) count as new records for the state.
\end{abstract}

Keywords: Diversity; Amazon; Fauna.

\section{Topic: Notas Científicas}

Reviewed anonymously in the process of blind peer
Received: 02/12/2020

Approved: 25/02/2021
Manoel Daltro Nunes Garcia Junior Universidade Federal do Amapá, Brasil http://lattes.cnpq.br/5420263758950354 http://orcid.org/0000-0002-7555-3414 m.d.juniorbio@gmail.com

Monique Telcia dos Santos Damasceno Universidade Federal do Amapá, Brasil http://lattes.cnpq.br/3198422156214271

http://orcid.org/0000-0003-0373-1389 telciamonique6@gmail.com

Raimundo Nonato Pincaço Souto (ic Universidade Federal do Amapá, Brasil http://lattes.cnpq.br/1661932002902797 http://orcid.org/0000-0002-8795-1217 rnpsouto@unifap.br
Referencing this:

GARCIA JÚNIOR, M. D. N.; DAMASCENO, M. T. S.; SOUTO, R. N. P.. Novos Registros da família Aeshnidae (Odonata: Anisoptera) para o estado do Amapá, Brasil. Nature and Conservation, v.14, n.1, p.181184, 2021. DOI: http://doi.org/10.6008/CBPC23182881.2021.001.0020 


\section{INTRODUÇÃO}

A ordem Odonata compreende os insetos popularmente conhecidos como libélulas, lava-bunda, entre outros. Atualmente existem aproximadamente 7.000 espécies descritas (KALKMAN et al., 2008). Na região neotropical ocorrem cerca de 1.800 espécies (VON ELLENRIEDER, 2009), para o Brasil são registradas em torno de 860 espécies (PINTO, 2018) o que representa cerca de $14 \%$ da fauna global.

Segundo Rehn (2003), a ordem Odonata está dividida em duas subordens: Zygoptera e Anisoptera, com 11 e cinco famílias ocorrentes no Brasil respectivamente (KOROIVA et al., 2020). Aeshnidae é uma família da subordem Anisoptera, apresenta ampla distribuição mundial, compreendendo 51 gêneros e aproximadamente 460 espécies descritas (DIJKSTRA et al., 2013). Nas Américas ocorrem cerca de 150 espécies inseridas em 20 gêneros (GARRISON et al., 2006). Para o Brasil, são assinaladas 60 espécies inseridas nos gêneros: Anax Leach in Brewester, 1815, Castoraeschna Calvert, 1952, Coryphaeschna Williamson, 1903, Gynacantha Rambur, 1842, Limnetron Förster, 1907, Neuraeschna Hagen, 1867, Remartinia Navás, 1911, Rhionaeschna Förster, 1909, Staurophlebia Brauer, 1865 e Triacanthagyna Selys, 1883. Com exceção do gênero Limnetron, que até o momento conta apenas com registros para as regiões Sul e Sudeste do país, todos os demais gêneros ocorrem na região Amazônica.

Os espécimes de Aeshnidae são geralmente indivíduos de grande porte, excelentes voadores, podendo atingir altas velocidades e longas distâncias, muitas espécies da família ainda possuem hábitos crepusculares (CORBET, 1999). Devido a essas características, geralmente trabalhos de levantamentos de Odonata resultam em um baixo número de capturas de exemplares da família Aeshnidae. Com isso, o objetivo deste trabalho é apresentar uma lista de espécies da família Aeshnidae, incluindo cinco novos registros de ocorrência para a fauna do estado do Amapá.

\section{MATERIAIS E MÉTODOS}

O estado do Amapá é um dos nove estados brasileiros que compõe a Amazônia legal, está localizado em uma área conhecida como escudo das Guianas (Figura 1), grande parte de seu território é composto por áreas protegidas (OLIVEIRA, 2010). De acordo com a classificação de Köppen Geiger o clima para o estado é do tipo Af (tropical superúmido), com temperatura média anual em torno de $27.0^{\circ} \mathrm{C}$, a pluviosidade média anual é de $3.300 \mathrm{~mm}$.

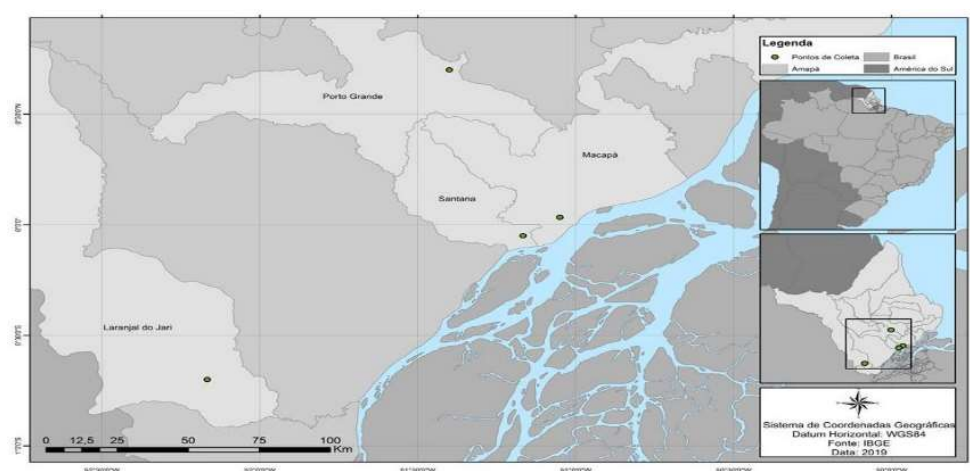

Figura 1: Mapa de distribuição dos pontos de coleta nos municípios de Laranjal do Jari, Macapá, Porto Grande e Santana, no estado do Amapá na região amazônica Brasil. 
As coletas de Odonata ocorreram durante o ano de 2018, em quatro municípios amapaenses, sendo

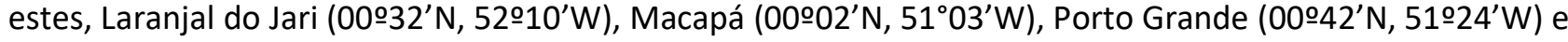
Santana $\left(00^{\circ} 03^{\prime} \mathrm{N}, 51010^{\prime} \mathrm{W}\right)$. Os exemplares foram capturados com auxílio de redes entomológicas. A identificação dos insetos foi realizada por meio de chaves taxonômicas propostas por Garrison et al. (2006) e Heckman (2006). Os Indivíduos capturados estão depositados na coleção científica do laboratório Arthropoda - ArthroLab da Universidade federal do Amapá- UNIFAP.

\section{RESULTADOS E DISCUSSÃO}

Durante as amostragens foram coletados 58 exemplares, pertencentes a sete espécies incluídas em cinco gêneros (Tabela 1). As espécies Gynacantha nervosa Rambur, 1842, Neuraeschna costalis Burmeister, 1839, Staurophlebia reticulata (Burmeister, 1839), Triacanthagyna ditzleri Williamson, 1923 e Triacanthagyna septima (Selys in Sagra, 1857) contam como novos registros para o estado do Amapá. O gênero Triacanthagyna $(n=24)$ compreendeu o maior número de espécimes coletados, seguido de Gynacantha $(n=17)$ e Coryphaeschna $(n=14)$.

Tabela 1: Lista das espécies de Aeshnidae coletados nos municípios de Laranjal do Jari (L), Macapá (MC), Porto Grande (PG) e Santana (SA) no estado do Amapá, Brasil. Os espécimes marcados com * são novos registros para o estado.

\begin{tabular}{l|l|l}
\hline Gênero & Espécies & Município \\
\hline Coryphaeschna Williamson, 1903 & & \\
\hline $\begin{array}{l}\text { Gynacantha } \\
\text { Rambur, 1842 }\end{array}$ & Coryphaeschna adnexa (Hagen, 1861) & MC,PG,SA \\
\hline & & \\
\hline & Gynacantha mexicana Selys, 1869 & LJ,MC,PG \\
\hline Neuraeschna Hagen, 1867 & Gynacantha nervosa Rambur, 1842* & PG \\
\hline $\begin{array}{l}\text { Staurophlebia } \\
\text { Brauer, 1865 }\end{array}$ & Neuraeschna costalis Burmeister, 1839* & PG \\
\hline & & \\
\hline $\begin{array}{l}\text { Triacanthagyna } \\
\text { Selys, 1883 }\end{array}$ & $\begin{array}{l}\text { Staurophlebia reticulata (Burmeister, } \\
\text { 1839* }\end{array}$ & LJ \\
\hline & & \\
\hline & $\begin{array}{l}\text { Triacanthagyna ditzleri Williamson, } \\
1923^{*}\end{array}$ & MC,PG, SA \\
\hline & $\begin{array}{l}\text { Triacanthagyna septima (Selys in Sagra, } \\
1857)^{*}\end{array}$ & MC,PG, SA \\
\hline
\end{tabular}

T. ditzleri foi a espécie com maior abundância com 17 exemplares coletados, seguida de G. mexicana e C. adnexa com 16 e 14 espécimes coletados respectivamente, essas espécies corresponderam a 81\% de todos os indivíduos amostrados durante o estudo. G. nervosa e $N$. costalis foram as espécies menos representativas, com apenas um exemplar cada uma. Os representantes da família Aeshnidae, possuem em sua maioria hábitos crepusculares voando ao anoitecer e ao amanhecer (NEEDHAM et al., 2000), além dos seus hábitos de voo, que incluem a permanência dos indivíduos em grandes altitudes durante a maior parte do tempo, fato que explica o baixo número de exemplares encontrados na maioria dos trabalhos.

\section{CONCLUSÕES}

Levantamentos faunísticos de Odonata têm aumentado ultimamente em diferentes estados 
brasileiros, contudo, ainda é baixo o conhecimento sobre a ordem. Segundo Marco et al. (2005) apenas 29\% do território brasileiro apresentam dados referentes à biodiversidade do grupo, indicando que são necessários maiores estudos com a Odonatafauna. Os resultados aqui apresentados demostram que a diversidade ocorrente no estado do Amapá deve ser relativamente maior, de modo que, a partir de novos levantamentos outros registros podem ocorrer para o estado.

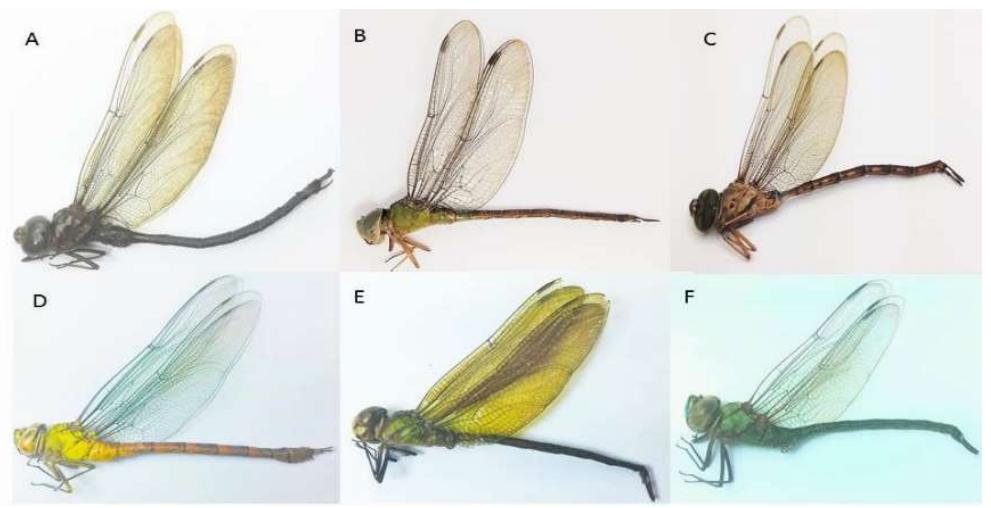

Figura 2: Imagens dos novos registros de Odonata para o estado do Amapá, fotos Manoel Garcia Jr. 2019. (A) Neuraeschna costalis Burmeister, 1839, (B) Triacanthagyna septima (Selys in Sagra, 1857), (C) Gynacantha nervosa Rambur, 1842, (D) Staurophlebia reticulata (Burmeister, 1839), (E) Triacanthagyna ditzleri Williamson, 1923, (F) Coryphaeschna adnexa (Hagen, 1861).

AGRADECIMENTOS: Este estudo foi financiado pela CNPq (Conselho Nacional de Desenvolvimento Científico e Tecnológico), e FAPEAP (Fundação de Amparo à Pesquisa do Estado do Amapá).

\section{REFERÊNCIAS}

CORBET, P. S.. Dragonflies: Behavior and ecology of Odonata. Ithaca: Comstock Publishing Associates, Cornell University Press, 1999.

DIJKSTRA, K. D. B.; BECHLY, G.; BYBEE, S. M.; DOW, R. A.; DUMONT, H. J.; FLECK, G.; GARRISON, R. W.; HÄMALÄINEN, M.; KALKMAN, V. J.; KARUBE, H.; MAY, M. L.; ORR, A. G.; PAULSON, D. R.; REHN, A. C.; THEISCHINGER, G.; TRUEMAN, J. W. H.; VAN TOL, J.; VON ELLENRIEDER, N.; WARE, J.. The classification and diversity of dragonflies and damselflies (Odonata). In Animal biodiversity: an outline of higher-level classification and survey of taxonomic richness (Zhang Z.-Q. eds.). Zootaxa, v.3703, p.1-82, 2013.

GARRISON, R. W.; VON ELLENRIEDER, N.; LOUTON, J. A.. Dragonfly genera of the New World. An illustrated and annotated key to the Anisoptera. The Johns Hopkins University Press Baltimore. Maryland, 2006.

HECKMAN, C. W.. Encyclopedia of South American Aquatic Insects: Odonata - Anisoptera. Illustrated Keys to Known Families, Genera, and Species in South America. Netherlands, 2006.

KALKMAN, V. J.; CLAUSNITZER, V.; DIJKSTRA, K. D. B.; ORR, A. G.; PAULSON, D. R.; VAN TOL, J.. Global diversity of dragonflies (Odonata) in freshwater. Hydrobiologia, v.595, p.351-363, 2008.
KOROIVA, R.; NEISS, U. G.; FLECK, G.; HAMADA, N.. Checklist of dragonflies and damselflies (Insecta: Odonata) of the Amazonas state, Brazil. Biota Neotrop, v.20, n.1, e20190877, 2020. DOI: https://doi.org/10.1590/1676-0611-bn-2019$\underline{0877}$

MARCO JUNIOR, P.; VIANNA D. M.. Distribuição do esforço de coleta de Odonata no Brasil subsídios para escolha de áreas prioritárias para levantamentos faunísticos. Lundiana, v.6, p.13-26, 2005.

NEEDHAM, J. G.; WESTFALL JUNIOR, M. J.; MAY, M. L. Dragonflies of North America. Scientific Publishers, Gainsville, 2000.

OLIVEIRA, M. J.. Diagnóstico do setor mineral do Estado do Amapá. Macapá: Instituto de Pesquisas Cientificas e Tecnológicas do Estado do Amapá, 2010.

PINTO, A. P.. Odonata in catálogo taxonômico da fauna do Brasil. PNUD, 2018.

REHN, A. C.. Phylogenetic analysis of higher-level relationships of Odonata. Systematic Entomology, v.28, p.181-239, 2003.

VON ELLENRIEDER, N.. Databasing dragonflies: state of knowledge in the Neotropical region. Agrion, v.13, p.58-72, 2009.

A CBPC - Companhia Brasileira de Produção Científica (CNPJ: 11.221.422/0001-03) detém os direitos materiais desta publicação. Os direitos referem-se à publicação do trabalho em qualquer parte do mundo, incluindo os direitos às renovações, expansões e disseminações da contribuição, bem como outros direitos subsidiários. Todos os trabalhos publicados eletronicamente poderão posteriormente ser publicados em coletâneas impressas sob coordenação da Sustenere Publishing, da Companhia Brasileira de Produção Científica e seus parceiros autorizados. Os (as) autores (as) preservam os direitos autorais, mas não têm permissão para a publicação da contribuição em outro meio, impresso ou digital, em português ou em tradução. 